\title{
High glucose conditions suppress the function of bone marrow-derived endothelial progenitor cells via inhibition of the eNOS-caveolin-1 complex
}

\author{
CHUNLI CAO ${ }^{1}$, HONGKUN ZHANG $^{2}$, LI GONG $^{1}$, YUNJUN HE $^{2}$ and NAN ZHANG ${ }^{1}$ \\ ${ }^{1}$ Department of Endocrinology, Sir Run Run Shaw Hospital, College of Medicine, \\ Zhejiang University, Hangzhou 310016; ${ }^{2}$ Department of Vascular Surgery, First Affiliated Hospital, \\ College of Medicine, Zhejiang University, Hangzhou 310003, P.R. China
}

Received July 22, 2011; Accepted September 27, 2011

DOI: $10.3892 / \mathrm{mmr} .2011 .644$

\begin{abstract}
The present study aimed to reveal how high glucose affects rat bone marrow-derived endothelial progenitor cells in vitro. Total mononuclear cells of bone marrow were obtained, cultured in endothelial cell growth medium-2 and identified by fluorescence microscopy. Using immunofluorescence, endothelial progenitor cells were identified by expression of VEGFR-2 as well as CD133 and were further characterized as those adherent cells which were double positive by Dil-Ac-LDL uptake and FITC-UEA-1 lectin binding. The attached cells were collected and glucose was added to the culture medium at various final concentrations $(11.1,33.3$ and $55.5 \mathrm{mmol} / \mathrm{l})$. Proliferation, migration and in vitro angiogenic ability of endothelial progenitor cells were measured. The change in mRNA and protein levels of caveolin-1 and endothelial nitric oxide synthase (eNOS) were examined; in addition, nitric oxide (NO) levels in the cell medium were measured. Based on the results, with increasing glucose concentration in the medium, proliferation, migration and in vitro angiogenic capacity of the cells were reduced, whereas mRNA and protein levels of caveolin-1 gene increased gradually. The mRNA levels of the eNOS gene did not differ, but the protein expression was reduced and NO levels in the culture medium declined. In conclusion, high glucose conditions are detrimental to the function of bone marrow-derived endothelial progenitor cells in vitro, probably by damaging the eNOS-caveolin-1 complex, which results in the reduction of $\mathrm{NO}$ synthesis, eventually leading to the impaired function of endothelial progenitor cells.
\end{abstract}

Correspondence to: Dr Nan Zhang, Department of Endocrinology, Sir Run Run Shaw Hospital, College of Medicine, Zhejiang University, No. 3 East Qingchun Road, Hangzhou 310016, P.R. China

E-mail: doczkm@yahoo.com.cn

Key words: diabetes mellitus, high glucose, endothelial progenitor cells, eNOS-caveolin-1 complex

\section{Introduction}

Patients in the late stage of type 2 diabetes frequently suffer from macrovascular complications, including coronary heart disease, cerebrovascular disease and peripheral macrovascular complications. Amputation caused by peripheral macrovascular complications could significantly increase the risk of death and reduce patients' quality of life. Endothelial progenitor cells (EPCs) are precursor cells and have been identified in the bone marrow, umbilical cord blood and peripheral blood. EPCs have the characteristics of mobility, migration, homing to the ischemic tissue, secreting growth factors, differentiating into endothelial cells and involvement in angiogenesis in the ischemic areas (1). Recent studies have shown that EPCs have a reduced capacity for regeneration, adhesion and migration, and contribute to the occurrence and development of peripheral vascular disease in diabetic patients. Decreased activity of EPCs from peripheral blood and bone marrow may be related to hyperglycemia, hyperinsulinemia, duration of diabetes, high cholesterol and other factors in diabetes $(2,3)$.

It has been established that endothelial nitric oxide synthase (eNOS) plays a key role in vascular relaxation by catalyzing cells to produce nitric oxide (NO). Moreover, caveolin is the surface marker and structure protein of caveolae, which are independent areas in the cell membrane. It is an essential molecule for the normal physiological function of the cardiovascular system. Caveolin-l has been identified as one of the caveolin family members (4). One study has found that eNOS is situated in caveolae, combines with caveolin-1 and forms the complex of eNOS-caveolin-1, which is closely associated with the homing and angiogenetic properties of endothelial cells (5).

In this study, we investigated the effect of high glucose on bone marrow-derived EPCs and the function of the eNOS-caveolin-1 complex, and their relationship was further determined. Our results revealed a potential therapeutic target for diabetes with vascular complications.

\section{Materials and methods}

Animal experiments. Male SD rats, 8-10 weeks old and weighing $250-280 \mathrm{~g}$, were used for the experiments. Animal 
experiments were approved by the Animal Ethics Committee of Zhejiang University.

Isolation of bone marrow-derived mononuclear cells (BM-MNCs) and EPCs culture. Rat bone marrow cells were isolated from the femora and tibiae. Mononuclear cells were separated by suspending the cells in Ficoll-Paque solution and centrifuged at $2000 \mathrm{rpm}$ for $25 \mathrm{~min}$. After being washed with phosphate-buffered saline (PBS), cells were resuspended with endothelial cell growth medium-2 (EGM-2, Lonza, Switzerland) and inoculated into 6-well plates. The medium was changed every 3 days (6).

Immunofluorescence. When adherent cells reached 80-90\% confluency, they were digested and passaged to 24-well plates. Once fully adhered, the immunofluorescence technique was performed using primary antibodies of vascular endothelial growth factor receptor 2 (VEGFR2; Cell Signaling Technology, USA) and CD133 (Cell Signaling Technology, USA), both of which were expressed on EPCs, and secondary antibody of fluorescein-conjugated anti-rabbit $\operatorname{IgG}$ (Cell Signaling Technology, USA). Cells were visualized using an inverted fluorescence microscope (7).

Dil-Ac-LDL/lectin staining. Dil-Ac-LDL/lectin staining was performed to further confirm the EPCs. Cells were incubated in 24-well plates using medium with $10 \mu \mathrm{g} / \mathrm{ml}$ 1,1-dioctadecyl-3,3,3,3-tet-ramethylindocarbocyanine perchlorate-labeled acetylated low-density lipoprotein (Dil-Ac-LDL) Molecular Probes, Eugene, OR, USA) for $10 \mathrm{~h}$. Cells were then fixed and counterstained with $10 \mu \mathrm{g} / \mathrm{ml}$ FITC-labeled UEA-1 lectin (Vector, USA) for $1 \mathrm{~h}$. After being washed three times with PBS, the cells were observed under fluorescence microscopy and images were captured (8).

Cell treatment. When the primary cells reached $80-90 \%$ confluence, the adherent cells were trypsinized, resuspended and randomly divided into four groups as follows: control group (cells were cultured in EGM-2 medium without additional glucose), and treatment groups (comprising 3 groups; glucose was added to the medium and calculated to final concentrations of $11.1,33.3$ and $55.5 \mathrm{mmol} / \mathrm{l})$.

EPCs proliferation assay. The cells in each group described above were inoculated into 96-well plates at a density of $1 \times 10^{4}$ EPCs in $200 \mu \mathrm{l}$ EGM-2 per well and cultured for $48 \mathrm{~h}$. Then, $20 \mu \mathrm{l}$ of MTT liquid was added to each well. The supernatant was removed and $150 \mu \mathrm{l}$ dimethyl sulfoxide was added to each well $4 \mathrm{~h}$ later, followed by oscillations for $10 \mathrm{~min}$. The OD values of each group were then analyzed at a wavelength of $490 \mathrm{~nm}(9)$.

EPCs migration assay. The migration of EPCs was evaluated by Millicell-PCF (Millipore, MA, USA) assay. EPCs $\left(n=5 \times 10^{4}\right)$ in $200 \mu \mathrm{l} \mathrm{EGM-2} \mathrm{were} \mathrm{placed} \mathrm{in} \mathrm{the} \mathrm{upper} \mathrm{chamber} \mathrm{of} \mathrm{24-well}$ Millicell plates and $600 \mu \mathrm{l}$ medium containing recombinant vascular endothelial growth factor (VEGF, $50 \mathrm{ng} / \mathrm{ml}$ ) was placed in the lower chamber, followed by incubation for $24 \mathrm{~h}$. The membrane was washed with PBS and fixed. The upper side of the membrane was wiped and the membrane was stained using crystal violet solution. The magnitude of migration of EPCs was evaluated by counting the migrated cells in 6 random high-power microscopic fields (magnification, x200) (9).

EPCs tube formation assay. The tube formation assay was performed to assess the capacity for EPCs vasculogenesis. In brief, Matrigel (BD Biosciences, Franklin Lakes, NJ, USA) was mixed with serum-free EGM-2, and placed in a 96-well plate at $37^{\circ} \mathrm{C}$ for $30 \mathrm{~min}$ to allow solidification. EPCs $\left(\mathrm{n}=1 \times 10^{4}\right)$ in $100 \mu 1$ EGM- 2 were inoculated in the 96-well plate, and incubated at $37^{\circ} \mathrm{C}$ for $24 \mathrm{~h}$. Representative fields $(n=6)$ were taken and the average of the total area of complete tubes formed was compared using the Image-Pro Plus computer software. When a cell deformed to the extent that its length was 3 times greater than its width, it was considered as a small tube (10).

Real-time reverse transcriptase-polymerase chain reaction $(R T-P C R)$. After culturing for $48 \mathrm{~h}$, the cells of each group were immediately homogenized and purified. Purified RNA was quantified and converted to cDNA. The amplification protocol was as follows: $95^{\circ} \mathrm{C}$ for $30 \mathrm{~min}$, followed by 40 cycles at $95^{\circ} \mathrm{C}$ for $5 \mathrm{sec}, 60^{\circ} \mathrm{C}$ for $34 \mathrm{sec}$, and $72^{\circ} \mathrm{C}$ for $30 \mathrm{sec}$. The data acquisition was performed at $72^{\circ} \mathrm{C}$. PCR was performed using a gene-specific primer pair for rat GAPDH, eNOS and caveolin-1 (Sangon, Shanghai, China). The following primers were used: GAPDH forward, 5'-CAAGCCCAACAACAAGG-3, and reverse, 5'-TGGTTC CGCAATCACAT-3'; eNOS forward, 5'-CTGCTGCCCCAGAT ATCTTC-3'; reverse, 5'-CAGGTACTGCAGTCCCTCCT-3'; caveolin-1 forward, 5'-CAAGCCCAACAACAAGG-3'; reverse, 5'-TGGTTCCGCAATCACAT-3'. Gene expression was then determined by real-time RT-PCR and the absolute number of gene copies was quantified.

Western blot analysis. In each treatment group, cells were cultured in 6-well plates for $72 \mathrm{~h}$ and then collected. Cells were lysed, and $40 \mu \mathrm{g}$ total proteins was separated on SDS-polyacrylamide gels and transferred to polyvinylidine fluoride membranes. They were blocked for $2 \mathrm{~h}$ and incubated overnight at $4^{\circ} \mathrm{C}$ with primary antibodies. Primary antibodies used included rabbit anti-eNOS (1:200 in TBS-T, Santa Cruz, Santa Cruz, CA, USA), rabbit anti-caveolin-1 (1:1000 in TBS-T, Cell Signaling Technology, Danvers, MA USA) and rabbit anti- $\beta$-actin (1:2000 in TBS-T, Cell Signaling Technology) polyclonal antibody. Bound antibodies were visualized using secondary antibodies and enhanced chemiluminescence detection. The analytical gels contained control samples for internal signal correction (11).

NO levels. The cells treated as described above were seeded in 96-well plates at a density of $1 \times 10^{4}$ in $200 \mu \mathrm{l}$ EBM-2 per well and incubated for $72 \mathrm{~h}$. The concentration of nitrite/nitrate ions $\left(\mathrm{NO}_{2}{ }^{-} / \mathrm{NO}_{3}{ }^{-}\right)$in the supernatant was then analyzed and compared with the standard well to calculate NO secretion. Each group included three duplicate wells.

Statistical analysis. All results were expressed as mean \pm SEM. Statistical analysis was performed with the Student's t-test for comparisons between two groups and 


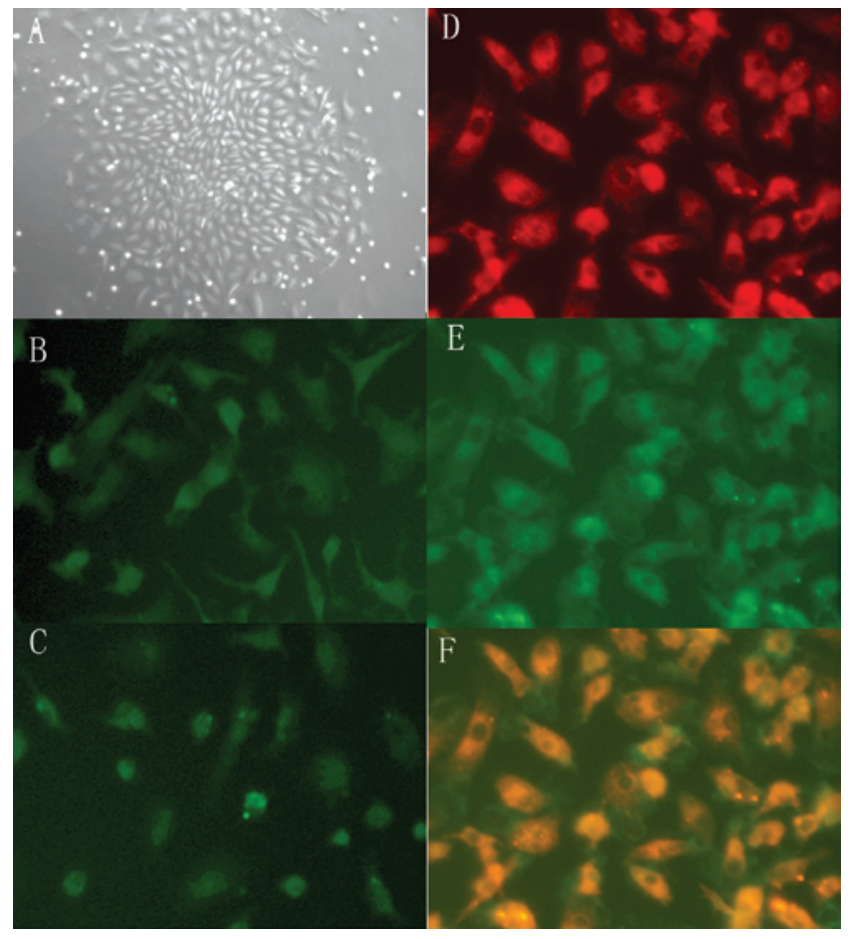

Figure 1. EPCs culture and identification. (A) Adherent cells were arranged in a spindle-like and colony-like manner. (B) Adherent cells expressed VEGFR-2. (C) Adherent cells expressed CD133. (D) Adherent cells were positive for uptake of Dil-Ac-LDL; red fluorescence was mainly noted in the cytoplasm. (E) Adherent cells exhibited positive binding of UEA-1-lectin; green fluorescence was mainly concentrated in the membrane. (F) Adherent cells were positive for uptake of Dil-Ac-LDL and binding of UEA-1-lectin. The double-positive cells were recognized as EPCs.

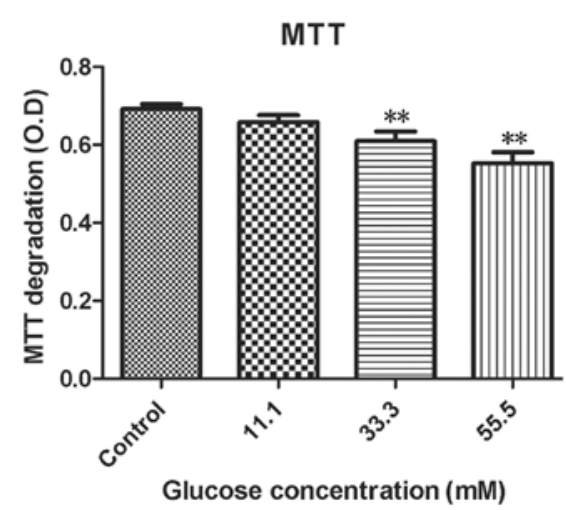

Figure 2. Influence of various glucose concentrations on the proliferation of EPCs. The greater the OD value, the stronger the cell proliferation. The OD value declined gradually in a manner dependent on the glucose concentration, indicating that the cell proliferation decreased. ${ }^{* *} \mathrm{p}<0.01$, significantly different from the control group.

ANOVA for more than two groups. $\mathrm{p}<0.05$ was considered statistically significant.

\section{Results}

EPCs culture and identification. In the case of the separated mononuclear cells cultured for 7-10 days, the majority of the cells were arranged in a fusiform, colony-like manner. As
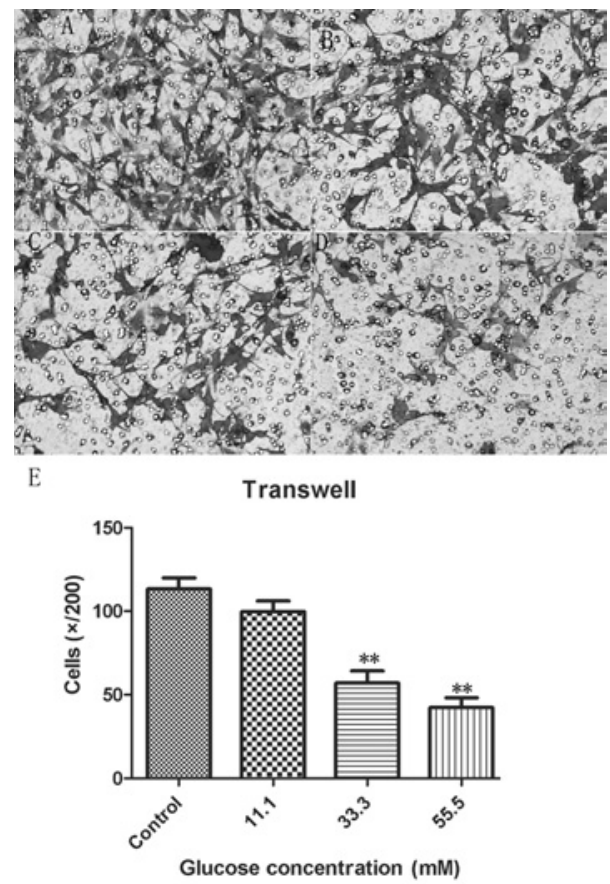

Figure 3. Influence of different glucose concentrations on the migration of endothelial progenitor cells (EPCs). These figures show migration of EPCs cultured in medium with various glucose concentrations: (A) control, (B) $11.1 \mathrm{mmol} / 1$, (C) $33.3 \mathrm{mmol} / 1$, (D) $55.5 \mathrm{mmol} / 1$, respectively. (E) The graph shows the mean values \pm SEM of four groups. The migratory capability of EPCs decreased, dependent on the increasing concentration of glucose. ${ }^{* *} \mathrm{p}<0.01$, significantly different from the control group.

shown in Fig. 1 and identified by immunofluorescence, $>85 \%$ of the cells expressed VEGFR-2 and $>80 \%$ expressed CD133. Furthermore, using Dil-Ac-LDL and FITC-UEA-1 lectin double-fluorescence staining, the double-positive cells were revealed to be endothelial progenitor cells.

High glucose suppresses the proliferation of bone marrow-derived EPCs. MTT was used to analyze EPCs proliferation. As shown in Fig. 2, the proliferation of EPCs decreased, according to the increase in glucose concentration. Although the difference between $11.1 \mathrm{mmol} / 1$ glucose $(0.6583 \pm 0.01796)$ and the control group $(0.6924 \pm 0.01186)$ were not statistically significant, the proliferation of EPCs was significantly inhibited by $33.3 \mathrm{mmol} / 1(0.6099 \pm 0.02417$ versus control, $\mathrm{p}<0.01)$ and $55.5 \mathrm{mmol} / \mathrm{l}$ glucose $(0.5530 \pm 0.02812$ versus control, $\mathrm{p}<0.01)$ compared with the control group.

High glucose suppresses the migration of bone marrow-derived EPCs. The modified millicell chamber assay was used to analyze EPCs migration ability. Fig. 3 shows that compared with the control group (113.3 \pm 6.556$), 11.1 \mathrm{mmol} / \mathrm{l}$ glucose $(96.67 \pm 6.433)$ did not significantly inhibit migration of EPCs, whereas $33.3 \mathrm{mmol} / 1(57.0 \pm 7.312$ vs. control, $\mathrm{p}<0.01)$ and $55.5 \mathrm{mmol} / 1$ glucose $(42.33 \pm 5.852$ vs. control, $\mathrm{p}<0.01)$ significantly inhibited the migration of EPCs.

High glucose suppresses the angiogenic ability of bone marrow-derived EPCs. Angiogenic ability of EPCs was examined by tube formation assay. As shown in Fig. 4, compared with the control group (28.67 \pm 1.282$)$, all treatment 


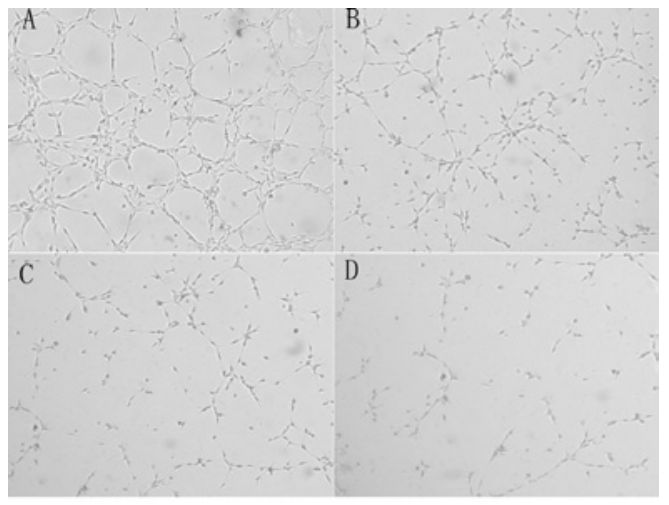

E

Tubes

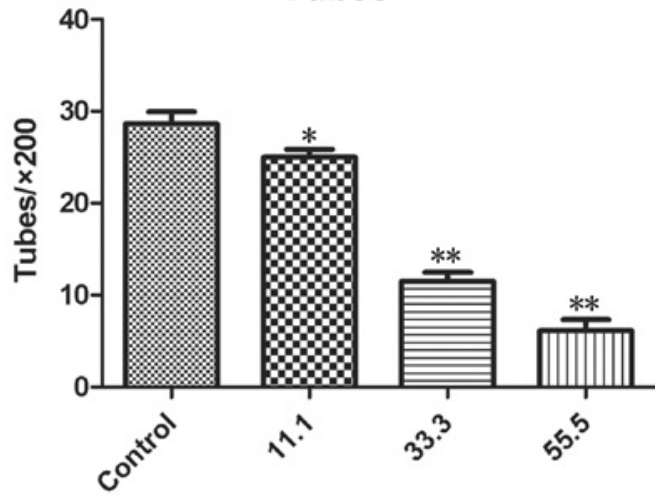

Glucose concentration (mM)

Figure 4. Influence of different glucose concentrations on the angiogenesis of EPCs. This figure shows the tube formation of EPCs cultured in medium with various concentrations of glucose: (A) control, (B) $11.1 \mathrm{mmol} / \mathrm{l},(\mathrm{C})$ $33.3 \mathrm{mmol} / \mathrm{l}$, (D) $55.5 \mathrm{mmol} / \mathrm{l}$, respectively. (E) The graph shows the mean values \pm SEM of the control and treated groups. The tubes formed by EPCs decreased in number in a concentration-dependent manner. ${ }^{*} \mathrm{p}<0.05,{ }^{* *} \mathrm{p}<0.01$, significantly different from the control group.

groups significantly inhibited the angiogenic capacity of EPCs (11.1 mmol/l glucose, $\mathrm{p}<0.05$ vs. control; $33.3 \mathrm{mmol} / 1$ and $55.5 \mathrm{mmol} / 1$ glucose, $\mathrm{p}<0.01$ vs. control).

High glucose affects gene expression of eNOS and caveolin-1 in various degrees. As observed using PCR and Western blot analysis, with the increasing glucose concentration, the mRNA level of the caveolin-1 gene and its protein expression increased gradually (Figs. 5 and 6). However, the protein expression of eNOS gradually declined, although its mRNA levels showed no significant change.

High glucose suppresses the secretion of NO. As shown in Fig. 7, in the presence of various concentrations of glucose, the NO concentration in the medium declined gradually in a concentration-dependent manner, and the difference was statistically significant compared with the control group.

\section{Discussion}

Since Asahara et al successfully demonstrated the existence of endothelial progenitor cells in 1997, increasing attention has been paid to EPCs (1). EPCs, which express surface markers including VEGFR-2 and CD133 and differentiate
A

\section{Caveolin-1/GAPDH}

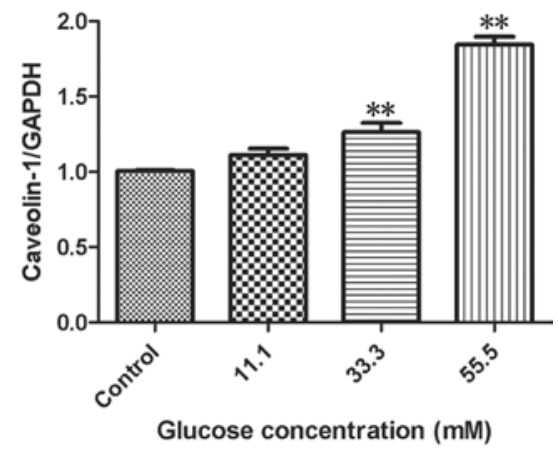

B

eNOS/GAPDH

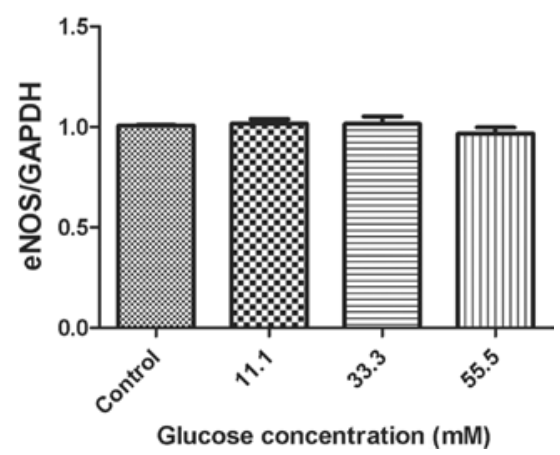

Figure 5. Caveolin-1 and endothelial eNOS mRNA expression in EPCs. mRNA levels of target genes were evaluated using real-time PCR, with GAPDH as internal control. Levels of target gene mRNAs were normalized to the level of GAPDH mRNA and the values were expressed relative to those of the control group. (A) In the presence of glucose, the mRNA level of caveolin-1 increased gradually in a concentration-dependent manner. (B) However, with regard to the eNOS mRNA levels, treated groups remained consistent as compared with the control group. ${ }^{* *} \mathrm{p}<0.01$, significantly different from the control group.

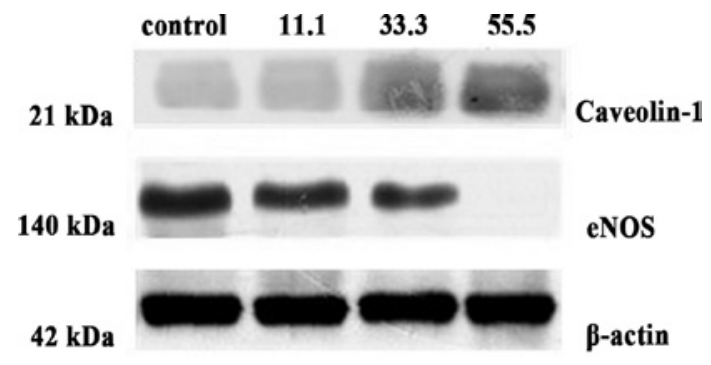

Figure 6. Caveolin-1 and endothelial nitric oxide synthase (eNOS) protein expression in EPCs. In response to increases in glucose concentration the protein expression of caveolin-1 gene increased whereas eNOS gene decreased gradually.

into endothelial cells, have the characteristics of mobility, migration, homing to the ischemic tissue, secreting growth factors and involvement in angiogenesis in ischemic areas. In 1999, Takeshita and Isner put forward the concept known as 'therapeutic angiogenesis', referring to the practice of complementing the number of EPCs or, through genetic modification of EPCs to improve their functions, rendering EPCs into the substrate of angiogenic factors and achieving more favorable angiogenic effects (12). EPCs transplantation in diabetic 
NO concentration

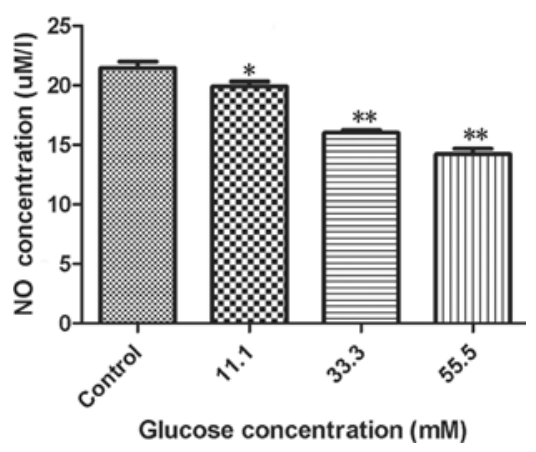

Figure 7. Nitric oxide (NO) levels in the medium. NO concentration in the medium decreased gradually in response to the rising level of glucose. The difference was statistically significant compared to the control group. " $\mathrm{p}<0.05,{ }^{* *} \mathrm{p}<0.01$, significantly different from the control group.

peripheral vascular disease, coronary heart disease, myocardial infarction and other ischemic diseases has shown marked potential for clinical application $(13,14)$.

However, certain studies have found that diabetes reduces the number of circulating EPCs and impairs their function, weakening the adhesion, migration and regeneration of EPCs (2). In their study, which used EPCs transplantation to treat severe limb ischemia in mice, Capla et al found that this method was significantly more effective in mice with simple peripheral vascular disease than in those with diabetes mellitus and peripheral vascular disease (15). The impact of diabetes on EPCs may have a number of causes, due to the complicated internal environment of the diabetic, but hyperglycemia is undoubtedly a highly significant aspect. High glucose in vitro was therefore used in our study to examine the influence of high glucose on the functions of bone marrowderived EPCs. We found that the proliferation, migration and angiogenesis of EPCs declined in response to the increase in glucose concentration. As glucose concentration increased, the proliferation of EPCs decreased. At the concentration of $11.1 \mathrm{mmol} / 1$ glucose, the proliferation was weaker than that of the control, but with no significant difference. However, the $33.3 \mathrm{mmol} / \mathrm{l}$ and $55.5 \mathrm{mmol} / \mathrm{l}$ groups were significantly different from the control group. High glucose also impaired angiogenic ability and migration of EPCs in a concentrationdependent manner. We therefore conclude that high glucose suppresses the proliferation, migration and angiogenesis of bone marrow-derived EPCs, and that this inhibition is concentration-dependent.

McGinn et al found that high glucose inhibits the proliferation of endothelial cells through the p38 mitogen-activated protein kinase (p38 MAPK) pathway (16). Kuki et al revealed that a high glucose concentration inhibits cell proliferation and promotes the phosphorylation of p38 MAPK, and the application of p38 MAPK inhibitor restores cell proliferation, indicating that the MAPK pathway may play a significant role in the damage caused by high glucose. However, increased glucose concentrations induce dysfunction of several intracellular signal transduction cascades, including modulation of protein kinase $\mathrm{C}$, generation of reactive oxygen species, and accumulation of advanced glycation end product (17).
Therefore, there may be other manners in which high glucose influences EPCs.

eNOS is one of the most vital enzymes determining the endothelial function. eNOS catalyzes the production of NO, a relaxing factor, which determines the function of endothelial cells and is essential for vascular formation and migration of endothelial cells (18). Caveolae, independent areas in the cell membrane, are abundant in the cardiovascular system and are involved in various physiological processes. Caveolin is a surface marker protein and structure protein of caveolae. Caveolin-1 a member of the caveolin family is abundant in endothelial cells. In a study on caveolin-1 knockout mice, Wunderlich et al found that disruption of caveolin-1 led to enhanced nitrosative stress as well as systolic and diastolic heart failure, which demonstrated that caveolin-1 plays a crucial physiological role in the cardiovascular system (19). As mentioned above, eNOS is situated in the caveolar membrane and binds to caveolin-1 forming a complex. Caveolin-1 interacts with reductase domains of eNOS and inhibit NO synthesis (20). Therefore, alteration of caveolin-1 abundance and its interaction with eNOS impact eNOS function. Our study found that, caveolin-1 protein increased, whereas eNOS protein decreased gradually, in response to the rising glucose concentration; moreover, in response to the the increase in glucose concentration, mRNA of caveolin-1 gene showed a gradual increase, while the eNOS gene did not, indicating that the caveolin-1 gene performs its regulatory role at the transcriptional level, whereas eNOS may do so at the posttranscriptional level. As mentioned above, NO produced by eNOS is an essential factor in the regulation of endothelial function and blood flow. We speculated that the variation in eNOS protein expression led to the differences in the production of NO, eventually leading to the impaired function of EPCs. In our study, just as we expected, we found that the the level of NO in the medium decreased in a concentration -dependent manner in response to the increase in glucose concentration, whereas the proliferation, migration and angiogenic ability of EPCs in vitro were gradually inhibited. These results confirmed our hypothesis.

However, there were several limitations to our study. Our evidence that high glucose impairs the function of EPCs partly through regulating caveolin-1 and eNOS expression was indirect. Additionally, it is unclear whether the study would be equally effective if we treated the diabetic lower limb ischemic mice with EPCs that are caveolin-1 downregulated or eNOS up-regulated. Further research on this subject is warranted.

In conclusion, to the best of our knowledge, our study was the first to demonstrate that, high glucose conditions suppress the proliferation, migration and in vitro angiogenic ability of bone marrow-derived EPCs via injury of the eNOS-caveolin-1 complex. This study may provide a new therapeutic target for diabetic patients with vascular complication.

\section{Acknowledgements}

This study was supported by the National Natural Science Foundation of China $(30700300,30300335)$, the Special Foundation for Young Talents of Zhejiang Municipal Bureau of Health (2009QN016) and Zhejiang Natural Science 
Foundation for Team Project of Distinguished Young Scholars (R2090377).

\section{References}

1. Asahara T, Murohara T, Sullivan A, et al: Isolation of putative progenitor endothelial cells for angiogenesis. Science 275: 964-967, 1997.

2. Ruiter MS, van Golde JM, Schaper NC, et al: Diabetes impairs arteriogenesis in the peripheral circulation: review of molecular mechanisms. Clin Sci 119: 225-238, 2010.

3. Albiero M, Menegazzo L, Boscaro E, et al: Defective recruitment, survival and proliferation of bone marrow-derived progenitor cells at sites of delayed diabetic wound healing in mice. Diabetologia 54: 945-953, 2011

4. Pan YM, Yao YZ, Zhu ZH, et al: Caveolin-1 is important for nitric oxide-mediated angiogenesis in fibrin gels with human umbilical vein endothelial cells. Acta Pharmacol Sin 27: 1567-1574, 2006.

5. Feron $\mathrm{O}$ and Balligand JL: Caveolins and the regulation of endothelial nitric oxide synthase in the heart. Cardiovasc Res 69: 788-797, 2006.

6. Kahler CM, Wechselberger J, Hilbe W, et al: Peripheral infusion of rat bone marrow derived endothelial progenitor cells leads to homing in acute lung injury. Respir Res 8: 50, 2007.

7. Friedrich EB, Walenta K, Scharlau J, et al: CD $34 / \mathrm{CD}^{-133^{+} /}$ VEGFR-2 $2^{+}$endothelial progenitor cell subpopulation with potent vasoregenerative capacities. Circ Res 98: e20-e25, 2006.

8. Benameur T, Tual-Chalot S, Andriantsitohaina R, et al: PPAR $\alpha$ is essential for microparticle-induced differentiation of mouse bone marrow-derived endothelial progenitor cells and angiogenesis. PLoS One 5: e12392, 2010.

9. Ii M, Takenaka H, Asai J, et al: Endothelial progenitor thrombospondin-1 mediates diabetes-induced delay in reendothelialization following arterial injury. Circ Res 98: 697-704, 2006.

10. Naruse K, Hamada Y, Nakashima E, et al: Therapeutic neovascularization using cord blood-derived endothelial progenitor cells for diabetic neuropathy. Diabetes 54: 1823-1828, 2005. Erratum in: Diabetes 55: 1534, 2006.
11. Zeng R, Chen YC, Zeng Z, et al: Effect of mini-tyrosyl-tRNA synthetase/mini-tryptophanyl-tRNA synthetase on ischemic angiogenesis in rats: proliferation and migration of endothelial cells. Heart Vessels 26: 69-80, 2011.

12. Takeshita S and Isner JM: Peripheral angiogenesis: therapeutic angiogenesis for peripheral vascular occlusive disease. Curr Interv Cardiol Rep 1: 147-156, 1999.

13. Chang HW, Leu S, Sun CK, et al: Level and value of circulating endothelial progenitor cells in patients with acute myocardial infarction undergoing primary coronary angioplasty: in vivo and in vitro studies. Transl Res 156: 251-263, 2010.

14. Comerota AJ, Link A, Douville J, et al: Upper extremity ischemia treated with tissue repair cells from adult bone marrow. J Vasc Surg 52: 723-729, 2010.

15. Capla JM, Grogan RH, Callaghan MJ, et al: Diabetes impairs endothelial progenitor cell-mediated blood vessel formation in response to hypoxia. Plast Reconstr Surg 119: 59-70, 2007.

16. McGinn S, Saad S, Poronnik P and Pollock CA: High glucosemediated effects on endothelial cell proliferation occur via p38 MAP kinase. Am J Physiol Endocrinol Metab 285: E708-E717, 2003.

17. Kuki S, Imanishi T, Kobayashi K, et al: Hyperglycemia accelerated endothelial progenitor cell senescence via the activation of p38 mitogen-activated protein kinase. Circ J 70: 1076-1081, 2006.

18. Dranka BP, Hill BG and Darley-Usmar VM: Mitochondrial reserve capacity in endothelial cells: the impact of nitric oxide and reactive oxygen species. Free Radic Biol Med 48: 905-914, 2010.

19. Wunderlich C, Schober K, Lange SA, et al: Disruption of caveolin-1 leads to enhanced nitrosative stress and severe systolic and diastolic heart failure. Biochem Biophys Res Commun 340: 702-708, 2006.

20. Ghosh S, Gachhui R, Crooks C, et al: Interaction between caveolin-1 and the reductase domain of endothelial nitricoxide synthase. Consequences for catalysis. J Biol Chem 273: 22267-22271, 1998. 